\title{
Mucinous tubular and spindle cell carcinoma with a high nuclear grade and micropapillary pattern: A case report
}

\author{
TORU SAKATANI $^{1 *}$, YOSHINAGA OKUMURA $^{1 *}$, NAOTO KURODA $^{2}$, TOSHIHIRO MAGARIBUCHI ${ }^{1}$, \\ YORIKA NAKANO ${ }^{3}$, TOSHIAKI SHIRAHASE $^{1}$, JUN WATANABE $^{1}$, YOJI TAKI ${ }^{1}$, \\ MITSUHIKO OKIGAKI ${ }^{4}$, SUSUMU IKEHARA ${ }^{5}$ and YASUSHI ADACHI ${ }^{6}$ \\ ${ }^{1}$ Department of Urology, Toyooka Hospital, Toyooka, Hyogo 668-8501; ${ }^{2}$ Department of Diagnostic Pathology, \\ Kochi Red Cross Hospital, Kochi 780-8562; ${ }^{3}$ Department of Histopathology and Cytology, Japanese Red Cross \\ Kyoto Daini Hospital, Kyoto 602-8026; ${ }^{4}$ Department of Internal Medicine, Otokoyama Hospital, \\ Yawata, Kyoto 614-8366; ${ }^{5}$ Professor Emeritus, Kansai Medical University, Hirakata, Osaka 573-1010; \\ ${ }^{6}$ Department of Diagnostic Pathology, Toyooka Hospital, Toyooka, Hyogo 668-8501, Japan
}

Received May 25, 2017; Accepted July 4, 2017

DOI: $10.3892 / \mathrm{mco} .2017 .1430$

\begin{abstract}
Mucinous tubular and spindle cell carcinoma (MTSCC) is rare in renal cell carcinoma (RCC) and usually demonstrates a low nuclear grade and a better prognosis compared with other RCCs. The authors present a case report of MTSCC containing an area of Fuhrman nuclear grade 3, in addition to an area with a micropapillary pattern. An 82-year-old man consulted a hospital due to macrohematuria, and a tumor in the right kidney was detected. The tumor was resected and histologically examined. The tumor consisted of various growth patterns: Elongated tubular structure, a papillary structure containing a micropapillary pattern and solid pattern with spindle cells. The tumor cells demonstrated Fuhrman nuclear grades 2 and 3. Invasion into the lymph vessel and metastasis into the regional lymph node were observed. Thus, the tumor was diagnosed as a high grade MTSCC. Five months following resection, a computed tomography scan suggested metastasis of the tumor into the para-aortic lymph nodes and liver, and the patient succumbed to brain metastasis. When MTSCC of kidney is observed, careful histological observation is important to avoid missing a high nuclear grade area.
\end{abstract}

\section{Introduction}

Mucinous tubular and spindle cell carcinoma (MTSCC) of the kidney is a rare tumor, which has been integrated into the World

Correspondence to: Dr Yasushi Adachi, Department of Diagnostic Pathology, Toyooka Hospital, 1094 Tobera, Toyooka, Hyogo 668-8501, Japan

E-mail: adachiya250@gmail.com

${ }^{*}$ Contributed equally

Key words: renal cell carcinoma, mucinous tubular and spindle cell carcinoma, mucin-poor variant, micropapillary pattern, metastasis
Health Organization (WHO) classification since 2004 (1-3). MTSCC is histologically characterized by elongated tubular, cord-like architecture and cuboidal to spindle cells with low nuclear grade and myxoid/mucinous stroma (1,3). MTSCC usually consists of low nuclear grade tumor cells, and MTSCC with high nuclear grade (more than Fuhrman grade 3 ) is extremely rare $(4,5)$.

A micropapillary growth pattern in carcinoma was first reported for breast cancer (6). Currently, micropapillary growth pattern is well known in various carcinomas, such as lung adenocarcinoma (7), colorectal adenocarcinoma (8), gastric adenocarcinoma (9), and urothelial carcinoma (10). However, micropapillary growth pattern in renal cell carcinoma (RCC) is extremely rare (11).

In this study, we described a case of high nuclear grade MTSCC with micropapillary pattern and poor prognosis.

\section{Case report}

An 82-year-old man consulted a hospital because of macrohematuria. The patient had brain infarction, diabetes mellitus, and hypertension. The patient's family provided written informed consent to the publication of the case details and associated images. The Ethics Committee of the Toyooka Hospital (Hyogo, Japan) approved the study.

A dynamic computed tomography (CT) scan revealed a 21-mm diameter tumor in the superior pole of the right kidney (Fig. 1). The inside of the tumor was slightly enhanced in the arterial and venous phases on the dynamic CT, but did not show strong enhancement in the arterial phase as observed in clear cell RCC. The right kidney was resected, and the tumor was macroscopically and histologically examined.

Macroscopically, a 27x25x25 mm-sized tumor residing in the cortex, medulla, and pelvis of the superior pole of the right kidney was revealed (Fig. 2). The tumor consisted of whitish multi-nodules without fibrous capsule, and showed necrotic area and bleeding. Microscopically, the tumor consisted of various growth patterns: a papillary structure containing a 

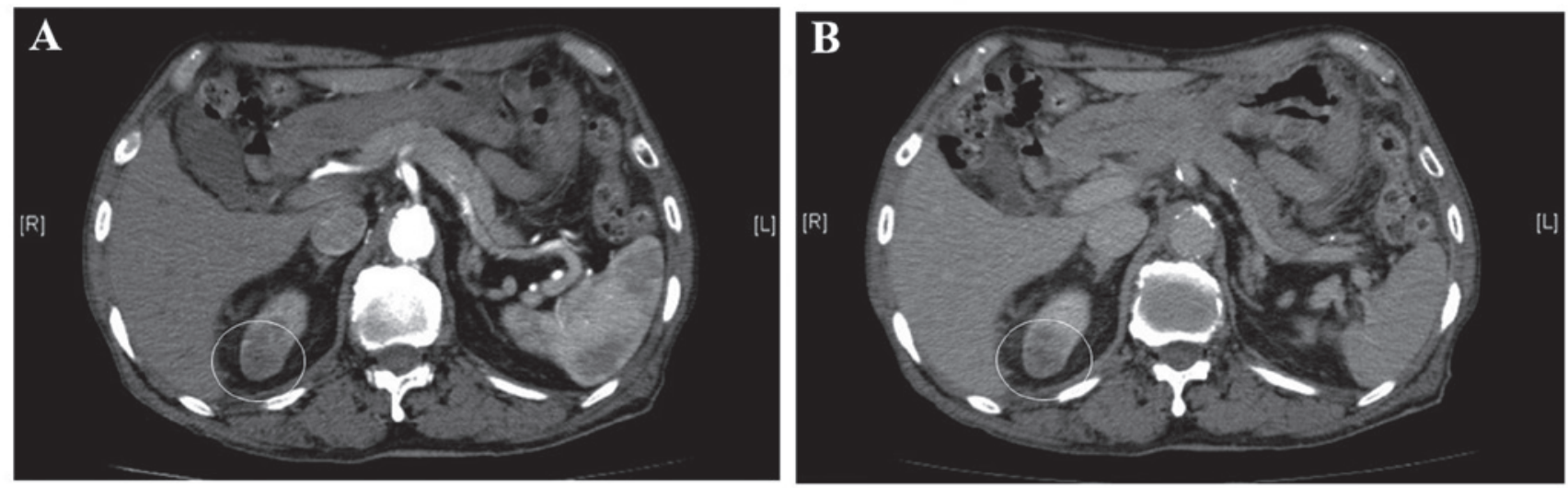

Figure 1. Dynamic computed tomography (CT) revealed a 21-mm diameter tumor in the superior pole of the right kidney. (A) Arterial phase image and (B) venous phase images of dynamic CT are shown. White-line circles show the tumor.
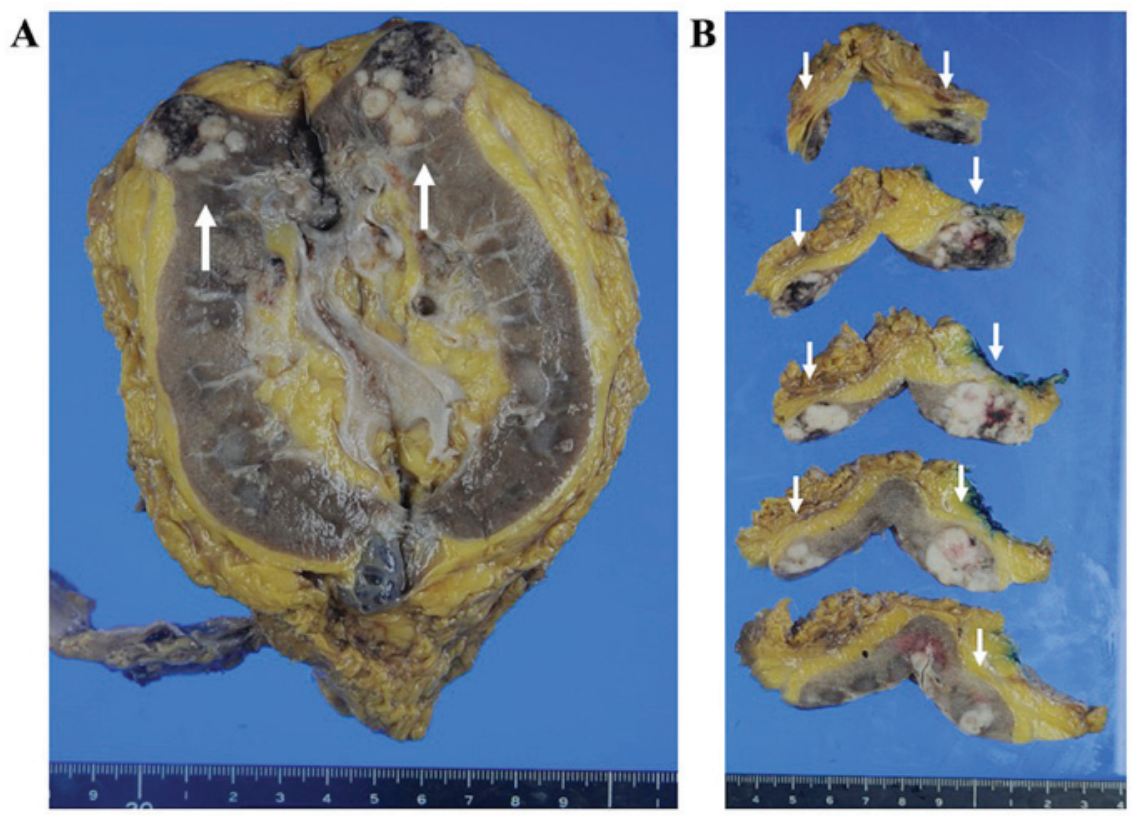

Figure 2. Macroscopical findings. (A) The sagittal cut surface of formalin-fixed resected kidney. (B) Horizontal cut surfaces of the kidney. Arrows show the tumor.

micropapillary pattern, an elongated tubular structure and, a solid pattern with spindle cells (Figs. 3 and 4). The tumor cells had eosinophilic cytoplasm. In the papillary area, two growth patterns of the tumor were observed: papillary growth with fibrovascular core and micropapillary pattern. We also found lymph vessel invasion of the MTSCC (Fig. 5). Invasion of the tumor into the lymph vessels was prominent in the micropapillary area in the MTSCC. In the small area, psammoma bodies were observed (data not shown). Small amounts of mucin were observed in the interstitium of the tumor using alcian blue stain. The tumor cells showed not only Fuhrman nuclear grade 2, but also Fuhrman nuclear grade 3. On immunohistological staining, the tumor was diffuse positive for cytokeratin 7, AMACR, and PAX8, partially positive for cytokeratin 19, PAX2, RCC Ma, and CD10, and negative for melanosome (HMB45), melan A, catepsin K, ALK, TFE3, and uroplakin II (Fig. 5, data not shown). In the micropapillary area, the tumor cells were positive for MUC-1 on the outside of the cell membrane (inside-out pattern), and EMA was positive on the cell membrane and cytoplasm of the tumor cells. Around the tumor were inflammatory cells mainly containing lymphoid cells and plasma cells, infiltrated with mild fibrosis. Solid growth with spindle and cuboidal cells with Fuhrman nuclear grade 3 were found in the regional lymph node, suggesting metastasis of MTSCC into the lymph node. Based on the morphological features, special staining and immunohistological staining, we diagnosed the tumor as MTSCC, mucin-poor variant with metastasis into the regional lymph node, and we also found a high nuclear grade area and micropapillary growth in the tumor.

Even after resection of the kidney, cytological examination of the patient's urine suggested malignancy. Four months after the operation, his urinary bladder was examined by cystoscopy, and transuretheral resection (TUR) was carried out. Urothelial carcinoma in situ was found in the TUR specimen.

Five months after resection of the kidney, follow-up positron-emission tomography (PET)-CT revealed swelling of the 

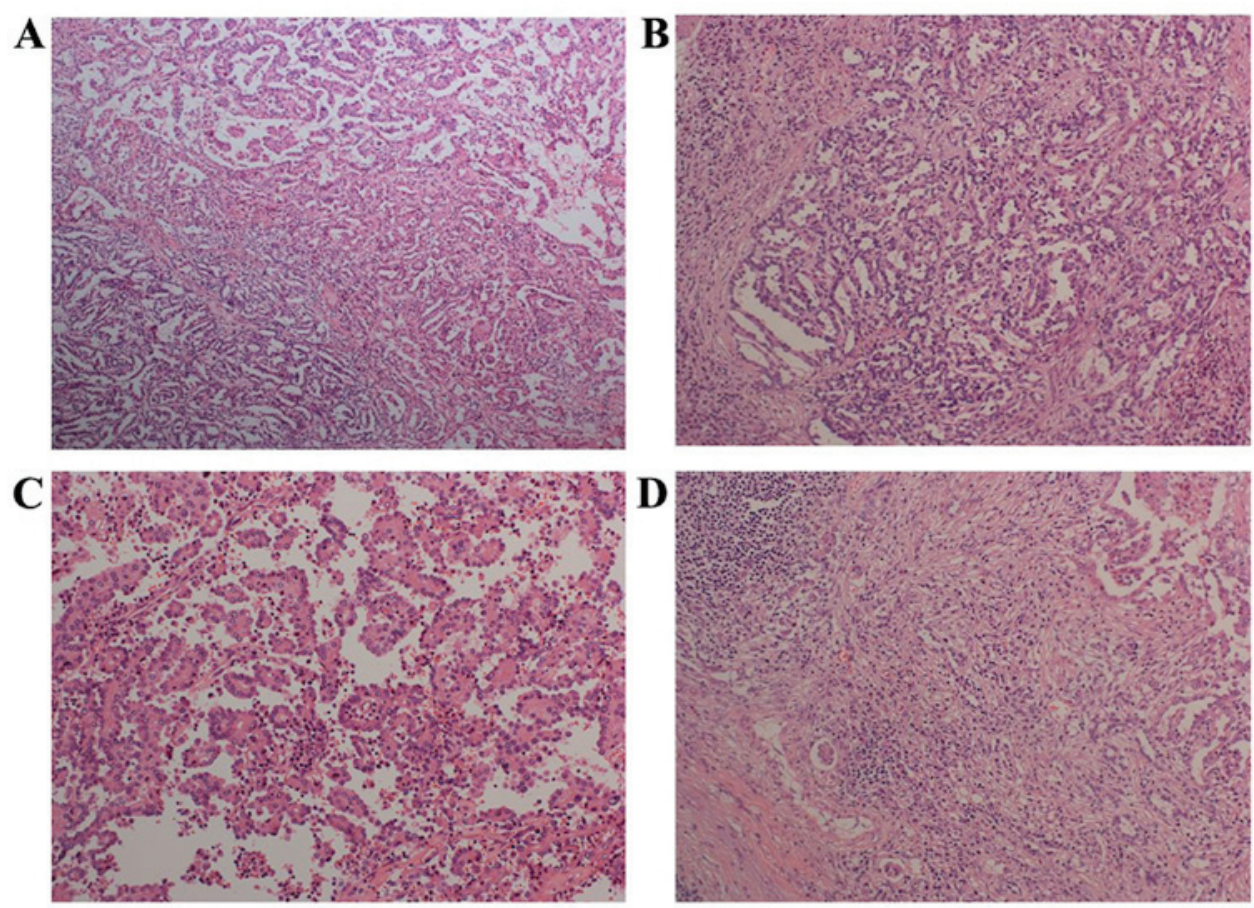

Figure 3. Microscopic findings of the tumor show various patterns of histology in hematoxylin and eosin staining. (A) Findings in the low power view (original objective lens: x4), (B) Tubular architecture, (C) papillary architecture, and (D) a solid pattern with spindle cells (original objective lens of B, C, and D: x20).

A

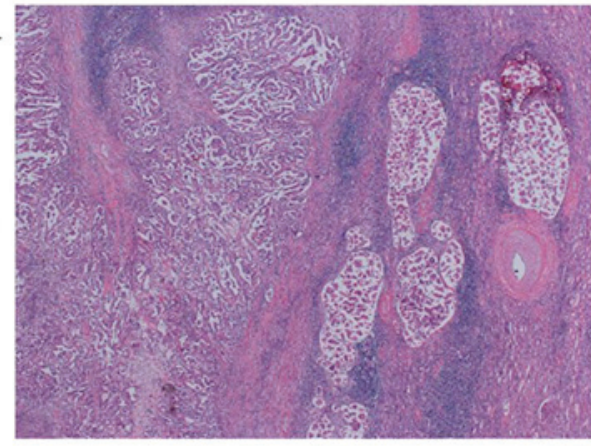

C

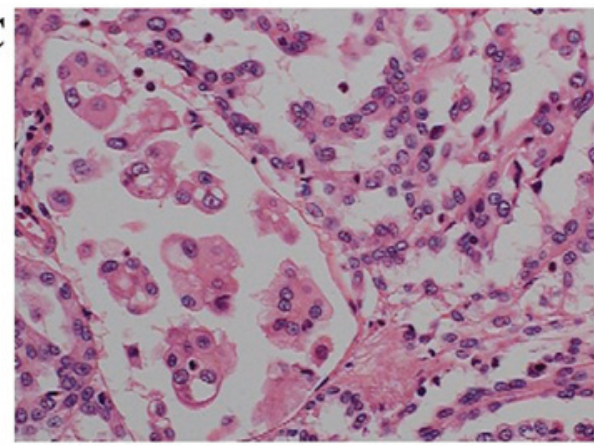

B

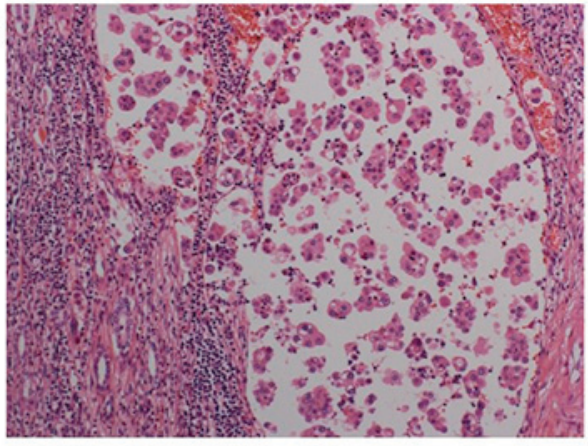

D

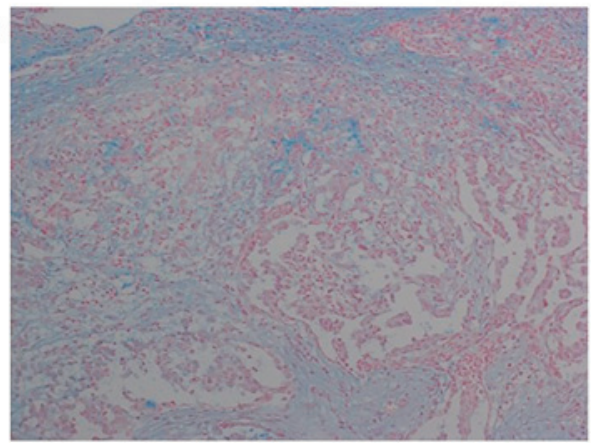

Figure 4. Micropapillary architecture in the tumor and mucin production of the tumor. (A) Papillary and micropapillary architecture of the tumor (objective lens: x4). (B) A middle-powered view of the micropapillary pattern of the tumor (objective lens: x20). (C) Tumor cells showing Fuhrman nuclear grades 2 or 3 (original objective lens: $\mathrm{x} 40$ ). (D) Mucin production of tumor cells detected by alcian blue staining (original magnification: x20).

para-aortic and para-common iliac arterial lymph nodes and a hepatic tumor, suggesting metastatic lesions of the MTSCC in the lymph nodes and liver. As another malignant tumor of the patient, urothelial carcinoma, was a carcinoma in situ, which usually do not metastasize. Administration of sunitinib was initiated to treat the metastatic lesions. Twelve days after the start of medication, cardiac and pulmonary arrest in the patient suddenly occurred. Cardiopulmonary resuscitation (CPR) was carried out on the patient. Brain CT revealed a brain hemorrhage and hydrocephalus, probably induced by intra-brain hemorrhage in the patient. The hemorrhage was thought to have occurred because of the metastatic lesion 

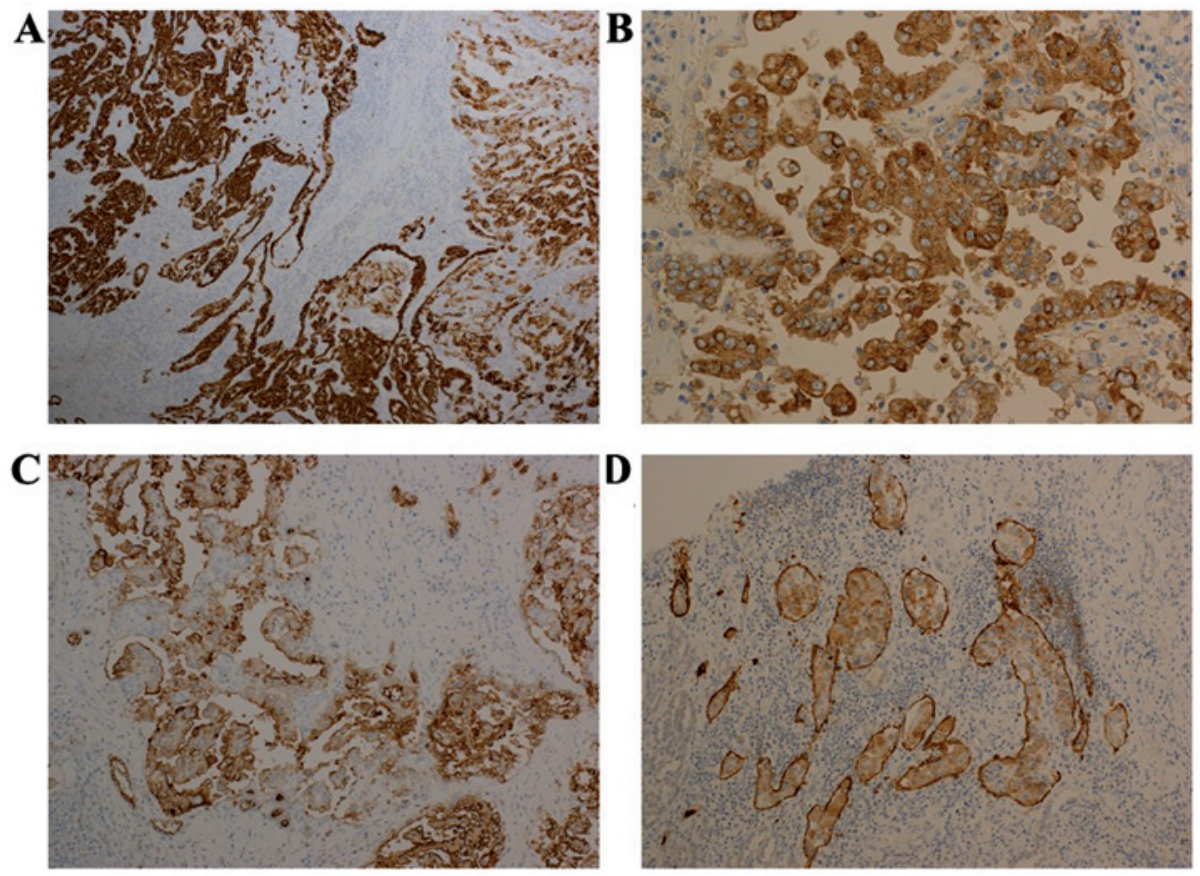

Figure 5. Immunohistochemical findings of the tumor. (A) The tumor is positive for CK7 (A) (original objective lens: x10). (B) In the area of micropapillary architecture, EMA was positive in the cytoplasm and cell membrane of the tumor cells. (C) MUC-1 was positive on the outside of the cell membrane of the tumor cells (original objective lens: x40). (D) Lymph vessels were positive for podoplanin, indicating invasion of the tumor into the lymph vessels (original objective lens: $\mathrm{x} 40$ ).

of the MTSCC. Although emergency external ventricular drainage was carried out, the patient succumbed to heart failure on the same day.

\section{Discussion}

MTSCC is a rare type of renal tumor, and MTSCC is believed to have been first reported in 1997 as 'low-grade collecting duct carcinoma' by MacLennan et al (12). The reported age range of MTSCC is between 17 and 82 years, with a mean age of 53 years and is dominant in female individuals $(13,14)$. In our case, the patient was an 82-year-old man, which is relatively rare in MTSCC cases with regard to age and gender. Although MTSCC is histologically characterized by elongated tubules and low-grade spindle cell components separated by pale mucinous stroma, mucin-poor variant of MTSCC has been also reported $(13,15-17)$. In our case, elongated tubules lined by cuboidal cells and a papillary pattern were prominent. However, spindle cells existed in certain areas containing a small amount of mucin. Therefore, we diagnosed our case as consistent with mucin-poor variant of MTSCC.

It has been reported that MTSCC is usually of a low nuclear grade and has a better prognosis than other RCCs (1). Rakozy et al have reported that patients with MTSCC show recurrences, but do not show distant metastasis or death by the tumor (2). However, MTSCC with a high nuclear grade (more than Fuhrman nuclear grade 3) has been reported, and it has also been reported that the prognosis for this minor MTSCC is not $\operatorname{good}(4,5,18)$.

In our case, invasion of the MTSCC into the lymph vessels and metastasis into the regional lymph node were found histologically. The metastasis of the MTSCC into the para-aortic lymph nodes, para-common iliac arterial lymph nodes, liver, and brain were suggested by diagnostic imaging, and the patient finally succumbed probably due to brain metastasis. These findings suggest that the MTSCC in our case was aggressive and had a poor prognosis. The MTSCC in our case contained not only a low nuclear grade area, but also a high nuclear grade one, suggesting that transformation from low to high grade could occur in MTSCC.

In our case, a part of the tumor showed micropapillary growth and lymph vessel-invasion of the micropapillary component. To the best of our knowledge, there has been only one report showing micropapillary growth in RCC (11). It has been reported that carcinomas showing micropapillary structure lead to a high incidence of lymph node metastasis (19) and poor prognosis (20-22). In our case, invasion into the lymph vessels in MTSCC was prominent in the area showing micropapillary structure. Moreover, we found that MTSCC showing micropapillary structure existed in the lymph vessels, suggesting that the micropapillary component could easily metastasize. Our patient also finally succumbed probably due to brain metastasis, and his MTSCC also showed high nuclear grade and micropapillary structure, suggesting a poor prognosis.

In this study, we have presented a case of mucin-poor MTSCC with high nuclear grade, micropapillary structure and metastasis into the lymph nodes and other organs. When diagnosing MTSCC, sufficient micro-slides should be prepared and observed carefully to detect the area of high nuclear grade.

\section{Acknowledgements}

We would like to thank Ms. H. Ogaki, Mr. K Nagaoka, Mr. T. Kuge, Mr. H. Takenaka and Ms. S. Eriguchi of the Toyooka Hospital for their expert technical assistance. 


\section{References}

1. Hes O, Hora M, Perez-Montiel DM, Suster S, Curík R, Sokol L, Ondic O, Mikulástík J, Betlach J, Peychl L, et al: Spindle and cuboidal renal cell carcinoma, a tumour having frequent association with nephrolithiasis: Report of 11 cases including a case with hybrid conventional renal cell carcinoma/spindle and cuboidal renal cell carcinoma components. Histopathology 41: 549-555, 2002.

2. Rakozy C, Schmahl GE, Bogner S and Störkel S: Low-grade tubular-mucinous renal neoplasms: Morphologic, immunohistochemical, and genetic features. Mod Pathol 15: 1162-1171, 2002.

3. Srigley J: Mucinous tubular and spindle cell carcinoma. IRAC Press, Lyon, 2004.

4. Shen SS, Ro JY, Tamboli P, Truong LD, Zhai Q, Jung SJ, Tibbs RG, Ordonez NG and Ayala AG: Mucinous tubular and spindle cell carcinoma of kidney is probably a variant of papillary renal cell carcinoma with spindle cell features. Ann Diagn Pathol 11: 13-21, 2007.

5. Kuroda N, Hes O, Michal M, Nemcova J, Gal V, Yamaguchi T, Kawada T, Imamura Y, Hayashi Y and Lee GH: Mucinous tubular and spindle cell carcinoma with Fuhrman nuclear grade 3: A histological, immunohistochemical, ultrastructural and FISH study. Histol Histopathol 23: 1517-1523, 2008.

6. Siriaunkgul S and Tavassoli FA: Invasive micropapillary carcinoma of the breast. Mod Pathol 6: 660-662, 1993.

7. Takanashi Y, Tajima S, Hayakawa T, Takahashi T, Neyatani H and Funai K: Pulmonary micropapillary-type adenosquamous carcinoma sharing epidermal growth factor receptor mutation in adenocarcinoma and squamous cell carcinoma. Respirol Case Rep 4: e00179, 2016.

8. Miyaoka Y, Fujiwara A, Kotani S, Tsukano K, Ogawa S, Yamanouchi S, Kusunoki R, Fujishiro H, Kohge N, Yamamoto T and Amano Y: Primary micropapillary carcinoma of the colon with submucosal invasion: A case report. Endosc Int Open 4: E744-E747, 2016.

9. Tanaka H, Baba Y, Sase T, Isono Y, Matsusaki S, Saito T, Okano H, Mukai K, Murata T and Watanabe G: Gastric intramucosal adenocarcinoma with an invasive micropapillary carcinoma component. Clin J Gastroenterol 8: 14-17, 2015.

10. Ishii S, Ohbu M, Toomine $\mathrm{Y}$, Nishimura $\mathrm{Y}$, Hattori $\mathrm{M}$, Yokoyama M, Toyonaga M, Kakinuma $\mathrm{H}$ and Matsumoto $\mathrm{K}$ : Immunohistochemical, molecular, and clinicopathological analyses of urothelial carcinoma, micropapillary variant. Pathol Int 61: 723-730, 2011.

11. Aoyagi T, Shinohara N, Kubota-Chikai K, Kuroda N and Nonomura K: Long-term survival in a patient with node-positive adult-onset Xp11.2 translocation renal cell carcinoma. Urol Int 86: 487-490, 2011.
12. MacLennan GT, Farrow GM and Bostwick DG: Low-grade collecting duct carcinoma of the kidney: Report of 13 cases of low-grade mucinous tubulocystic renal carcinoma of possible collecting duct origin. Urology 50: 679-684, 1997.

13. Fine SW, Argani P, DeMarzo AM, Delahunt B, Sebo TJ, Reuter VE and Epstein JI: Expanding the histologic spectrum of mucinous tubular and spindle cell carcinoma of the kidney. Am J Surg Pathol 30: 1554-1560, 2006.

14. Grigore A, Toma L, Stoicea M, Dinu M and Ardeleanu C: Rare renal tumor-mucinous tubular and spindle cell carcinoma. Rom J Morphol Embryol 53: 167-171, 2012.

15. Farghaly $\mathrm{H}$ : Mucin poor mucinous tubular and spindle cell carcinoma of the kidney, with nonclassic morphologic variant of spindle cell predominance and psammomatous calcification. Ann Diagn Pathol 16: 59-62, 2012.

16. Saito K, Shimada M, Inoue K, Shiiki K, Nagata M, Ogawa Y, Matsubara E, Maeda T, Matsumoto Y, Kunimura T and Mikogami T: A case of mucinous tubular and spindle cell carcinoma of the kidney. Hinyokika Kiyo 59: 107-111, 2013 (In Japanese).

17. Cao W, Huang B, Fei X, Huang X, Dai J, Zhou W, Xu Z, Su H, Cheng $K$ and Sun F: Clear cell changes in mucinous tubular and spindle cell carcinoma: Cytoplasmic pallor/clearing within tubules, vacuoles or hybrid conventional clear cell carcinoma of kidney? Int J Clin Exp Pathol 7: 4350-4358, 2014.

18. Arafah M and Zaidi SN: Mucinous tubular and spindle cell carcinoma of the kidney with sarcomatoid transformation. Saudi J Kidney Dis Transpl 24: 557-560, 2013.

19. Zekioglu O, Erhan Y, Ciris M, Bayramoglu H and Ozdemir N: Invasive micropapillary carcinoma of the breast: High incidence of lymph node metastasis with extranodal extension and its immunohistochemical profile compared with invasive ductal carcinoma. Histopathology 44: 18-23, 2004.

20. Amin MB, Tamboli P, Merchant SH, Ordóñez NG, Ro J, Ayala AG and Ro JY: Micropapillary component in lung adenocarcinoma: A distinctive histologic feature with possible prognostic significance. Am J Surg Pathol 26: 358-364, 2002.

21. Lee HJ, Eom DW, Kang GH, Han SH, Cheon GJ, Oh HS, Han KH, Ahn HJ, Jang HJ and Han MS: Colorectal micropapillary carcinomas are associated with poor prognosis and enriched in markers of stem cells. Mod Pathol 26: 1123-1131, 2013.

22. Bertz S, Wach S, Taubert H, Merten R, Krause FS, Schick S, Ott OJ, Weigert E, Dworak O, Rödel C, et al: Micropapillary morphology is an indicator of poor prognosis in patients with urothelial carcinoma treated with transurethral resection and radiochemotherapy. Virchows Arch 469: 339-344, 2016. 